\section{Correlation between peripapillary atrophy and corticosteroid therapy in patients with Vogt- Koyanagi-Harada disease}

${ }^{1}$ Singapore National Eye Centre, Singapore,

Singapore

${ }^{2}$ Division of Ophthalmology, Changi General Hospital,

Singapore, Singapore

${ }^{3}$ Singapore Eye Research Institute, Singapore, Singapore

${ }^{4}$ Department of Ophthalmology, National University of Singapore, Singapore, Singapore

Correspondence: S-P Chee, Singapore National Eye Centre, 11 Third Hospital Avenue, Singapore 168751 Singapore

Tel: + 6562277255 ;

Fax: +65 62277290 .

E-mail: chee.soon.phaik@

snec.com.sg

Received: 27 February 2006 Accepted in revised form: 8 August 2006

Published online:

15 September 2007

The Singapore Eye Research Institute was the sponsoring organization
Abstract

Aims To determine the correlation between systemic corticosteroid therapy and the occurrence and size of peripapillary atrophy (PPA) in patients with Vogt-Koyanagi-Harada (VKH) disease.

Methods All patients with VKH disease were retrospectively reviewed for their corticosteroid regimen. The extent of the PPA, if present, was measured using digitized imaging software, by two masked observers. Eyes with myopia greater than 6 dioptres or glaucoma were excluded. The patients were classified into three groups: early high (EH), late high (LH), and low dose (LD), according to the dose and timing of corticosteroids received during the acute phase of the disease.

Results There were 40 eyes in the EH group, 25 eyes in the LH group, and 23 eyes in the LD group. Multivariate analysis showed that corticosteroid therapy was the main determinant of PPA occurrence. All the eyes in the LD group had PPA and eyes in the LH groups were 4.02 times (95\% confidence interval 1.24-13.07) more likely to develop PPA than those in the EH group. The LD group also had larger PPA to disc ratios than the EH group. (Mean of 2.83 vs $0.19, P<0.001$ ) Conclusion The development and extent of PPA in patients with VKH disease appear to be dependent on the dose and timing of systemic corticosteroids.

Eye (2008) 22, 240-245; doi:10.1038/sj.eye.6702591; published online 15 September 2007

Keywords: Vogt-Koyanagi-Harada disease; corticosteroids
A Jap ${ }^{1,2}$, CD Luu ${ }^{3}$, I Yeo ${ }^{1}$ and S-P Chee ${ }^{1,3,4}$

Introduction

Peripapillary atrophy (PPA) is a nonspecific change seen in a number of uveitic diseases including Vogt-Koyanagi-Harada (VKH) disease, high myopes, and glaucoma patients. PPA area also increases with age. ${ }^{1}$

In VKH disease, there is an autoimmune reaction against the uveal melanocytes, with subsequent chorioretinal depigmentation giving rise to the characteristic 'sunset glow' fundus, and with chronic recurrent inflammation, chorioretinal atrophy may occur. The chorioretinal atrophy is usually diffuse, but it can also occur in a band around the optic disc (PPA) (Figure 1). In an earlier study we had found that $67 \%$ of our patients with VKH disease developed PPA during the course of their disease (Figure $2 \mathrm{a}$ and $\mathrm{b}$ ). ${ }^{2}$

Before the 1990s, the role of high dose corticosteroids in the management of VKH was not well recognized in Singapore and the patients were mainly treated with topical corticosteroids or low doses (LDs) of oral corticosteroids during the acute phase, even in eyes with severe disease. However, from the early 1990s onwards, most VKH patients presenting during the acute phase were treated with at least $1 \mathrm{mg} / \mathrm{kg}$ body weight (BW) of prednisolone per day.

The size of the PPA in our patients appeared to be dependent on the amount of corticosteroids received during the acute phase of the disease. Hence we felt that PPA may develop in $\mathrm{VKH}$ patients as a result of inadequate suppression of the inflammation, which most often involves the peripapillary region in VKH. The aim of this study was to 


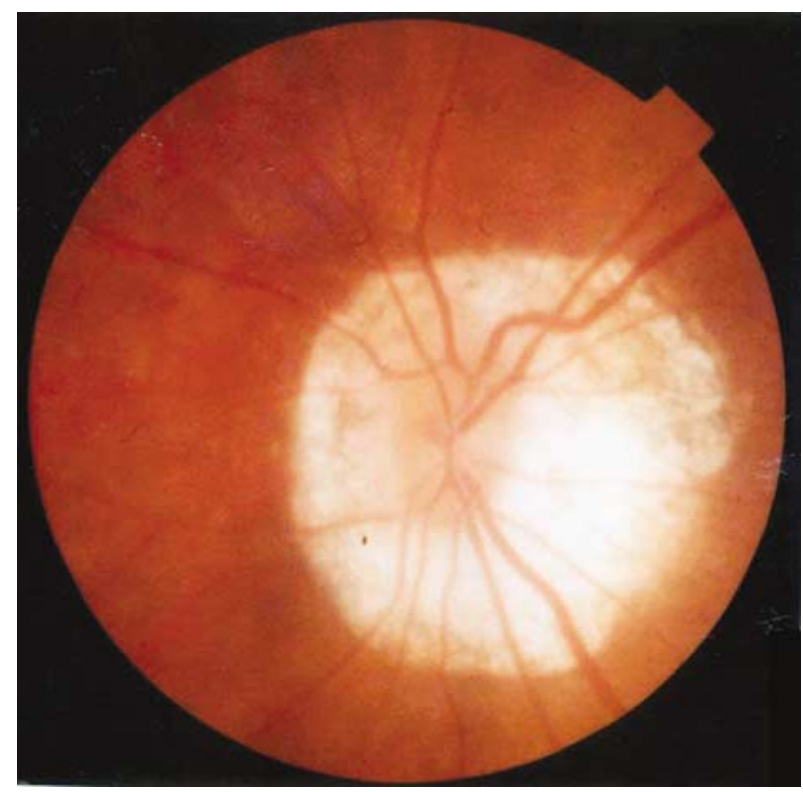

Figure 1 Disc photo of the right eye of a VKH patient in the LD group taken 10 years after onset of disease, showing a large area of PPA.

determine if there is an association between the amount of systemic corticosteroids received and the development as well as the size of the PPA.

\section{Materials and methods}

The records of all VKH patients, seen in the Uveitis Clinic of the Singapore National Eye Centre, from 1994 to 2002, were retrospectively reviewed for patient demographics, date of onset of symptoms, extent of ocular and/or extraocular involvement, date of initiation of treatment, dose and duration of corticosteroids received during the acute phase, duration of follow-up, refractive errors, presence of glaucoma, number of recurrent episodes, and the best-corrected Snellen acuity at onset and at the last visit.

The records reviewed included both our clinic notes as well as those of the referring physicians. The diagnosis of VKH was made according to the VKH Committee criteria. $^{3}$

Eyes with more than 6 dioptres of myopia or glaucomatous disc changes were excluded from analysis.

The disc photographs of eligible eyes were digitized and imported into the Imagenet system (Topcon Imagenet 2000 version 2.15, Tokyo, Japan). Only photographs that were of sufficient quality to enable the PPA and disc areas to be drawn accurately, taken when the disc oedema or hyperaemia had subsided, and within 6 months from the last follow-up visit, were included. Two masked observers measured the PPA and disc areas twice, using the Imagenet software, with at least $24 \mathrm{~h}$
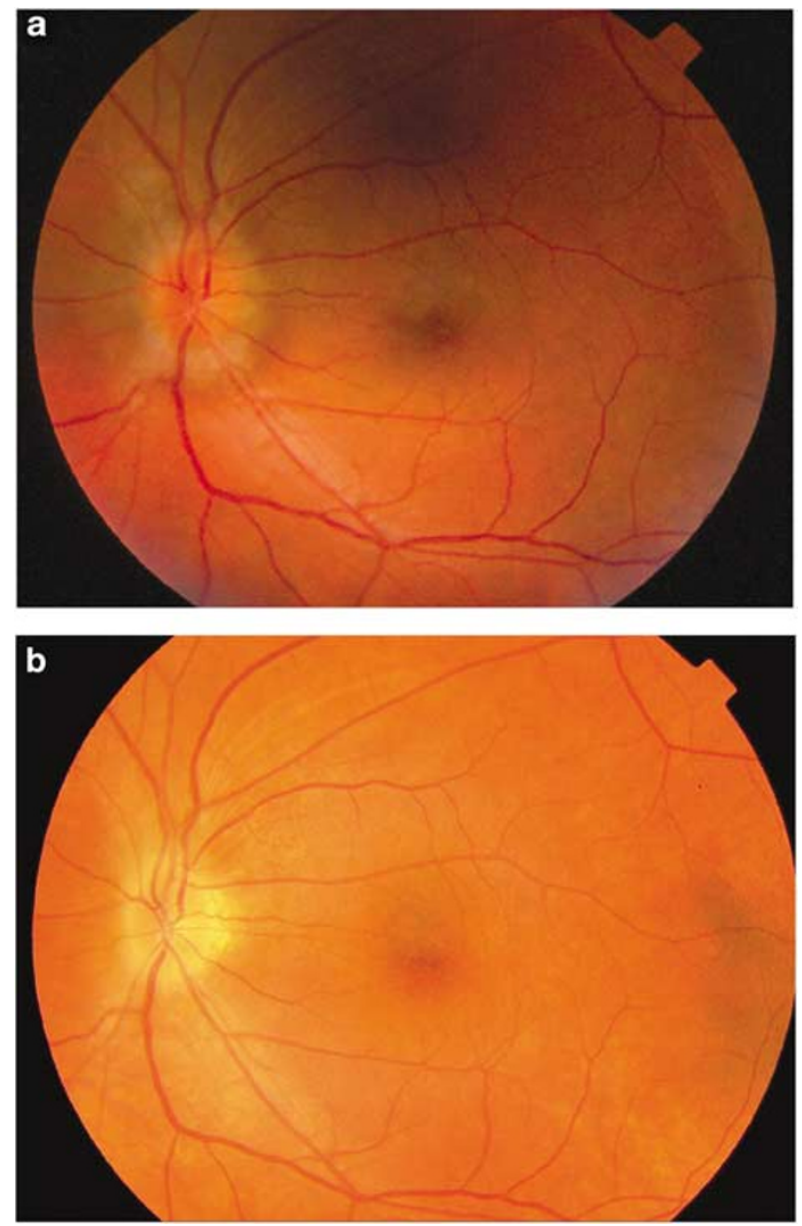

Figure 2 (a) Disc photo of the left eye of a patient with $\mathrm{VKH}$, taken 3 weeks after the onset of the acute phase, showing disc oedema. (b) Disc photo of same eye taken 7 months after onset, showing a small area of PPA. This patient had received a LH dose of steroids.

between the two measurements. Both zone alpha (a peripheral zone of irregular hypo- and hyperpigmentation) and zone beta (a central zone of chorioretinal atrophy with large visible choroidal and scleral vessels) were included in the PPA area. Elschnig's ring was not included in the PPA area. In order to eliminate the effects of refractive errors and the magnification of the photographs, the PPA to disc area ratio (PPA/D ratio) was calculated. The mean of the four values PPA/D ratio for each photograph was used for all subsequent analyses.

For purposes of analyses, the patients were divided into three groups, based on the time of initiation and dose of systemic corticosteroids received (either orally and/or intravenously) during the acute phase of their disease.

1. The early high (EH) group received at least $1 \mathrm{mg} / \mathrm{kg}$ BW per day of prednisolone starting from within 
2 weeks of onset of the acute phase and remained on systemic immunosuppressants for at least 3 months

2. The late high (LH) group received at least $1 \mathrm{mg} / \mathrm{kg}$ BW per day of prednisolone, with treatment started between 2 and 4 weeks after the onset of the acute phase and remained on systemic immunosuppressants for at least 3 months

3. The LD group consisted of those who did not receive any systemic corticosteroids, or received less than $1 \mathrm{mg} / \mathrm{kg} \mathrm{BW}$ per day of prednisolone or did not remain on systemic immunosuppressants for at least 3 months or received high dose prednisolone more than 4 weeks from the onset of the acute phase.

The duration of the disease refers to the interval between the onset of the disease and the time the disc photographs were taken.

Recurrences were defined as an increase in anterior chamber cells by at least $2+$ and/or an increase in vitreous haze grade on binocular indirect ophthalmoscopy of at least 1 .

Statistical analyses were performed using SPSS statistical software (SPSS version 10.0 Chicago, IL, USA).

\section{Results}

During the review period, 74 patients with VKH were managed in the Uveitis clinic. Eighty-eight eyes of $47(64 \%)$ of these patients satisfied the inclusion criteria. Only one photograph of each eye was of adequate quality for inclusion. Good disc photographs could not be obtained in one eye of six patients owing to small pupils and/or media opacity and were excluded from analysis. There were good inter-observer $(r=0.983, P<0.001)$ and intra-observer agreements for the PPA and disc measurements (Observer 1: $r=0.867, P<0.001$, Observer 2: $r=0.999, P<0.001$ ). In the 88 eyes examined, PPA was detected in 62 eyes $(70.45 \%)$.

The three treatment groups were fairly similar in terms of the age of onset of symptoms and racial distribution. There were slightly fewer males in the LD group. The LD group had a significantly longer duration of the disease than the EH group (Table 1). Of the 23 eyes in the LD group, one eye received oral prednisolone 2 days after onset of symptoms, at $1 \mathrm{mg} / \mathrm{kg} /$ day. However, the steroid dose was tapered very rapidly and discontinued by 3 weeks. Hence this eye was considered to have received LD steroids. The other 22 eyes received only topical and/or periocular dexamethasone injections in the first 3 months after onset of symptoms. Subsequently, three were treated with $1 \mathrm{mg} / \mathrm{kg} /$ day of prednisolone and another three were treated with $0.75 \mathrm{mg} / \mathrm{kg} /$ day of prednisolone.
Table 1 Demographic profile of the patients

\begin{tabular}{lcccc}
\hline & \multicolumn{4}{c}{ Steroid therapy } \\
\cline { 2 - 5 } $\begin{array}{l}\text { Demographic } \\
\text { profile }\end{array}$ & $\begin{array}{c}\text { Early high } \\
(\mathrm{n}=20)^{\mathrm{a}}\end{array}$ & $\begin{array}{c}\text { Late high } \\
(\mathrm{n}=13)^{\mathrm{a}}\end{array}$ & $\begin{array}{c}\text { Low dose steroids } \\
(\mathrm{n}=14)^{\mathrm{a}}\end{array}$ & P-value \\
\hline $\begin{array}{l}\text { Chinese race } \\
\text { Male sex }\end{array}$ & 16 & 11 & 13 & $\mathrm{NS}^{\mathrm{b}}$ \\
$\begin{array}{l}\text { Age at onset (years) } \\
\text { Mean }\end{array}$ & 9 & 5 & & $0.042^{\mathrm{b}}$ \\
$\begin{array}{l}\text { SD } \\
\text { Duration of disease (months) } \\
\text { Mean }\end{array}$ & 48 & 49 & 43 & $\mathrm{NS}^{\mathrm{c}}$ \\
SD & 13.07 & 14.34 & 14.13 & - \\
\hline
\end{tabular}

${ }^{a} \mathrm{n}$, number of patients.

${ }^{\mathrm{b}} \chi^{2}$ test; NS, no statistical significance at $5 \%$ significance level; SD, standard deviation.

'ANOVA.

The LD group had the largest proportion of eyes with PPA as well as the largest PPA/D ratios (Table 2).

Univariate logistic analysis showed that the duration of the disease and treatment type were significantly associated with the occurrence of PPA but not the sex or the age of onset (Table 3).

Multivariate logistic regression analysis showed that treatment group was the only determinant for the occurrence of PPA, and eyes in the LH group had 4.02 times (95\% confidence interval $=1.24-3.07$ ) higher risk of developing PPA than eyes in the EH group (Table 4).

Linear regression analysis of the PPA/D ratio vs duration of the disease showed a positive correlation between size of the PPA and duration in the LH group $(r=0.68, P<0.001)$. There was no correlation seen between the size of the PPA and duration for the other two treatment groups (Figure 3).

The EH group were less likely to have recurrences and had less frequent episodes than the LH and LD groups (Table 2).

There was no significant difference in the final bestcorrected visual acuity among the treatment groups (Table 2). Of the fifteen eyes with a final best-corrected visual acuity of worse than $6 / 12,10$ had poor vision owing to chorioretinal atrophy, one had subretinal fibrosis, one had chorioretinal neovascular membrane formation, and in the remaining three eyes, there were no clinically detectable abnormalities. Seven of the 10 eyes with chorioretinal atrophy were from the LD group, one was from the LH group, and two were from the $\mathrm{EH}$ group. The eye with chorioretinal neovascular membrane formation was from the LD group and had a PPA/D ratio of 7.25. The eye with subretinal fibrosis was from the EH group and had no PPA. 
Table 2 Steroid regimen and outcome measures

\begin{tabular}{lcccc}
\hline & \multicolumn{3}{c}{ Steroid therapy } \\
\cline { 2 - 5 } Outcome measure & $\begin{array}{c}\text { Early high } \\
(\mathrm{n}=40)^{\mathrm{a}}\end{array}$ & $\begin{array}{c}\text { Late high } \\
(\mathrm{n}=25)^{\mathrm{a}}\end{array}$ & $\begin{array}{c}\text { Low dose steroids } \\
(\mathrm{n}=23)^{\mathrm{a}}\end{array}$ & $\begin{array}{c}\text { P-value } \\
\text { Percentage of eyes with PPA }\end{array}$ \\
Mean PPA/D ratio & 47.5 & 80 & 100 & $<0.001^{\mathrm{b}}$ \\
SD & 0.19 & 1.29 & 2.83 & $<.99$ \\
Percentage of eyes with at least one episode of recurrences & 15 & 3.35 & 87 & $<0.001^{\mathrm{c}}$ \\
Mean recurrences/month & 0.0052 & 0.27 & 0.04 & 74 \\
SD & 0.016 & 0.054 & $0.001^{\mathrm{b}}$ \\
Percentage of eyes with BCVA of $6 / 12$ or better & 87.5 & 84 & $\mathrm{NS}^{\mathrm{b}}$ \\
\hline
\end{tabular}

${ }^{a} \mathrm{n}$, number of eyes.

${ }^{b} \chi^{2}$ test; NS, no statistical significance at 5\% significance level; SD, standard deviation.

'ANOVA.

Table 3 Univariate logistic regression analysis of risk factors for occurrence of PPA

\begin{tabular}{lcc}
\hline Risk factor & $\begin{array}{c}\text { Odds ratio } \\
(95 \% \text { confidence interval })\end{array}$ & P-value \\
\hline Duration of disease & $1.18(1.05-1.31)$ & 0.004 \\
Age at onset & $1.03(0.99-1.07)$ & 0.093 \\
Sex & & \\
$\quad$ Male & 1.0 & 0.179 \\
Female & $0.53(0.21-1.34)$ & - \\
Treatment type & & \\
EH & 1.0 & - \\
LH & $4.42(1.39-14.10)$ & 0.012 \\
LD & All eyes had PPA & - \\
\hline
\end{tabular}

Table 4 Multivariate analysis of risk factors for occurrence of PPA

\begin{tabular}{lcc}
\hline Risk factor & $\begin{array}{c}\text { Odds ratio } \\
(95 \% \text { confidence interval })\end{array}$ & P-value \\
\hline Duration of disease & $1.06(0.92-1.22)$ & 0.42 \\
Treatment type & 1 & \\
EH & $4.02(1.24-13.07)$ & 0.021 \\
LH & \\
\hline
\end{tabular}

\section{Discussion}

The occurrence of PPA was significantly influenced by the dose and timing of the corticosteroid received during the acute phase, with the highest proportion seen in eyes in the LD group and the lowest in the EH group.

This was a cross-sectional study with insufficient time points of PPA measurements available for each eye to enable us to study the effect of duration of disease on the size of PPA for individual eyes. However, plots of PPA vs duration of disease for the three treatment groups showed that there was a positive correlation between the size of the PPA and duration of the disease in the LH
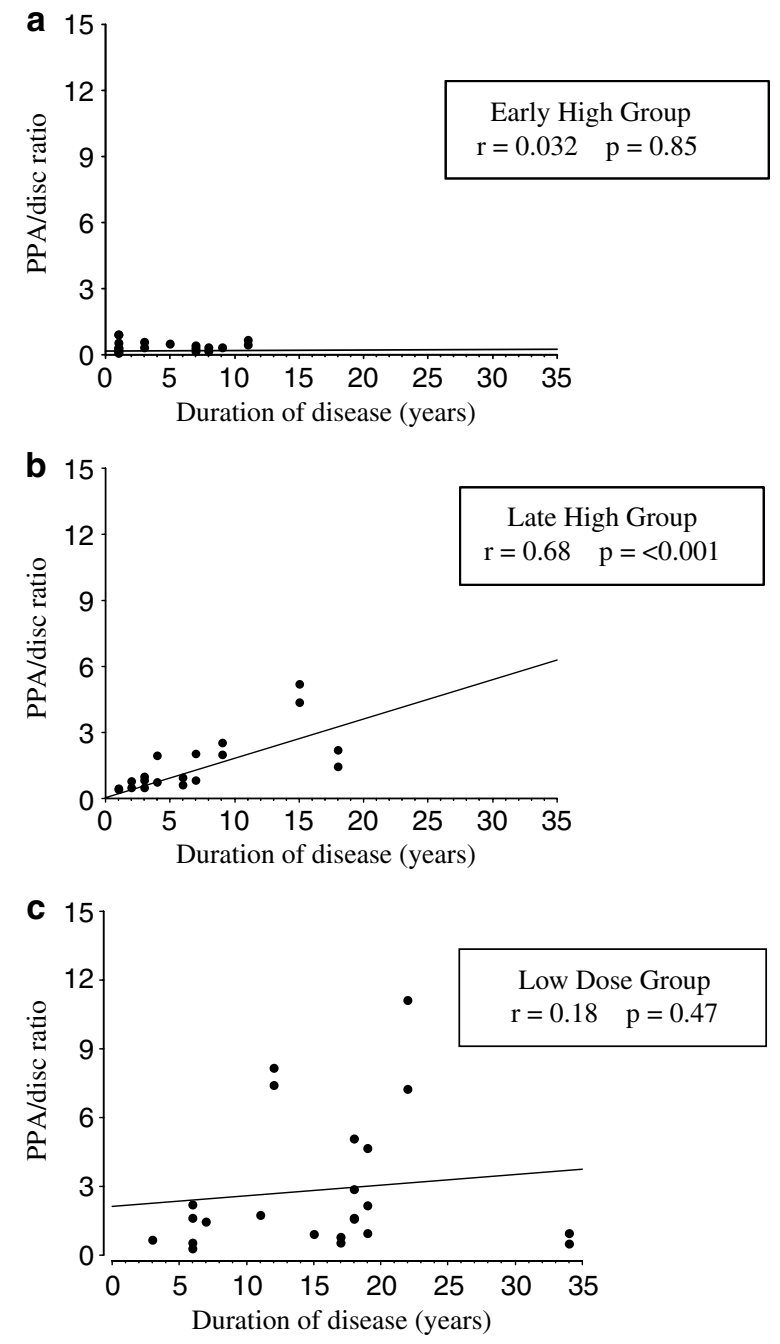

Figure 3 Graph of linear regression analysis of PPA/Disc ratio vs duration of disease PPA.

group. There was no correlation seen in the LD group between size and duration of disease owing to large variations in the treatment received by this group and in 
the duration of follow-up. Although no correlation was seen in the EH group, the graph suggests that the PPA/D ratios remained small up in this group, at least up to 15 years of follow-up.

Eyes in the LH and LD groups were more likely to have chronic disease as evidenced by the higher likelihood of recurrences as well as greater frequency of episodes of recurrences than eyes in the $\mathrm{EH}$ group. As these two groups also had longer durations of their disease, they would have had more total recurrent episodes of inflammation than the EH group. Indocyanine green angiographic studies of patients during episodes of recurrences showed choroidal changes suggestive of chronic ongoing inflammation, ${ }^{4,5}$ and this could contribute to the larger PPA in these two groups as well as the increase in size of the PPA over time in the LH group. The EH group had fewer recurrences and hence would have less ongoing choroidal inflammation and therefore their PPA were smaller and did not increase significantly in size over time. This suggests that while duration does influence the size of the PPA, its influence is partly a result of the inadequate initial control of inflammation.

There was no difference in the final Snellen acuity between the three treatment groups, probably owing to the small number of eyes with poor vision. However the main cause of poor vision in our patients was chorioretinal atrophy, and seven of these eyes were from the LD group. Moreover, in eyes with extensive chorioretinal atrophy, although they had good Snellen acuity, visual field defects, and abnormal electroretinograms have been noted. ${ }^{6}$ Similarly, we found markedly abnormal electroretinograms in eyes with PPA/D ratios greater than $2 .^{7}$ In addition, VKH patients who had only mild retinal atrophy also experienced visual disturbances ${ }^{5}$ and our patients with small PPA/D ratios also showed subclinical retinal dysfunction on electroretinography. ${ }^{7}$

In this retrospective study it was not possible to analyse whether the size of the PPA was related to the severity of the disease. However, the corticosteroid therapy received by these patients during the acute phase was determined mainly by prevailing medical practice patterns of the time, rather than by the severity of their disease. This is reflected in the fact the LD group has the longest duration of disease and the EH group the shortest, whereas if therapy had been dictated by severity, the duration of the disease would have been more similar in all groups. However as the patients were treated in blocks by time periods, each group would probably have had a spectrum of disease severity. Yet the LD group on the whole had larger PPAs than the EH group. In addition, PPA still occurred in almost half the eyes in the EH group. Hence our findings thus suggests that the development and the extent of PPA are largely determined by both the amount and timing of the immunosuppressant therapy.

Indocynaine green angiography ${ }^{4}$ has been proposed as a useful adjunct in the treatment of $\mathrm{VKH}$ patients and may enable earlier evaluation of the adequacy of the immunosuppression therapy. However, it is an invasive, costly, and time consuming procedure. Although the development of PPA is a relatively late sign, taking place at about 2-4 months after the signs of the acute phase has resolved, ${ }^{2}$ it can therefore still be useful as an additional measure of the adequacy of immunosuppressant therapy. It is also easier to assess clinically, even in eyes with small pupils, than other indicators of chronic inflammation such as chorioretinal depigmentation. If PPA is seen to occur after the acute phase, then it may be useful to perform multifocal electroretinography to evaluate the amount of inflammation at that time point and hence modify immunosuppressant therapy as appropriate. Its occurrence would also be an indicator that these patients will need closer monitoring for recurrences.

\section{Acknowledgements}

This work was presented in part as an oral presentation at the International Uveitis Study Group Meeting, Sydney, Australia April 2002 and at the IOIS Symposium, First SERI-ARVO Meeting on Research in Vision and Ophthalmology, Singapore, February 2003, as well as at 'Visual Function in Vogt-Koyanagi-Harada Disease' XVI International Congress of Eye Research, Sydney, 29 August-3 September 2004. The authors have no financial relationship with the Singapore Eye Research Institute that has sponsored the research, and have full control of all primary data and agree to allow Eye to review our data if requested.

\section{References}

1 Curcio CA, Saunders PL, Younger PW, Malek G. Peripapillary chorioretinal atrophy: Bruch's membrane changes and photoreceptor loss. Ophthalmology 2000; 107: 334-343.

2 Chee SP, Yeo YSI. Peripapillary atrophy in VogtKoyanagi-Harada syndrome. In: Ohno S, Aoki K, Usui M, Uchi E (eds). Uveitis Today - Excerpta Medica International Congress Series 1158. Elsevier: Amsterdam, 1998, pp 181-184.

3 Read RW, Holland GN, Rao NA, Tabbara KF, Ohno S, Arellanes-Garcia L et al. Revised diagnostic criteria for VogtKoyanagi-Harada disease: report of an international committee on nomenclature. Am J Ophthalmol 2001; 131(5): 647-652.

4 Bouchenaki N, Herbort CP. The contribution of indocyanine green angiography to the appraisal and management 
of Vogt-Koyanagi-Harada disease. Ophthalmology 2001; 108(1): 54-64.

5 Okada A, Mizusawa T, Sakai J, Usui M. Videofundoscopy and videoangiography using scanning laser ophthalmoscope in Vogt-Koyanagi-Harada syndrome. Br J Ophthamol 1998; 82: 1175-1181.

6 Sonoda S, Nakao K, Ohba N. Extensive chorioretinal atrophy in Vogt-Koyanagi-Harada disease. Jpn J Ophthalmol 1999; 43: 113-119.
7 Chee SP, Luu CD, Cheng CL, Lim WK, Jap A. Visual function in Vogt-Koyanagi-Harada patients. Graefes Arch Clin Exp Ophthalmol 2005; 243(8): 785-790.

8 Moorthy RS, Inomata H, Rao NA. Vogt-KoyanagiHarada syndrome. Surv Ophthalmol 1995; 39(4): 265-292.

9 Suzuki S. Quantitative evaluation of 'sunset glow' fundus in Vogt-Koyanagi-Harada disease. Jpn J Ophthalmol 1999; 43(4): 327-333. 